\title{
AN INDIRECT SUFFICIENCY PROOF FOR THE PROBLEM OF BOLZA IN NONPARAMETRIC FORM
}

\author{
BY \\ MAGNUS R. HESTENES
}

1. Introduction. Until recently sufficiency theorems in the calculus of variations have been established by direct methods, either by the construction of a Mayer field or by an expansion theorem. Indirect proofs have now been devised. The first indirect proof was given in 1942 by McShane[2]( $\left.{ }^{1}\right)$ who established a very general sufficiency theorem for a weak minimum in the problem of Bolza. The methods used by McShane were extended by Myers [3] so as to obtain a sufficiency theorem for a semistrong minimum in the nonparametric case. In certain special cases Myers obtained a strong sufficiency theorem. In a recent paper the author [6] showed the methods introduced by McShane can be modified so as to yield a strong sufficiency theorem for the parametric problem of Bolza. Although these results are phrased in terms of the parametric problem they are equally applicable to the nonparametric case. As yet the general sufficiency theorems given by McShane, Myers and the author have not been established by direct methods.

Although the sufficiency theorems given by the author are applicable to the nonparametric problem of Bolza by transforming this problem to a parametric one, the details of the method used do not appear to be sufficiently general to enable one to obtain an indirect proof of the sufficiency theorem for the nonparametric case without recourse to this transformation. It is the purpose of the present paper to remedy this situation and to obtain certain additional results that are of interest. The difficulties encountered earlier were overcome mainly by a suitable choice of a function $K\left(C, C_{0}\right)$ which measures the deviation of a comparison arc $C$ from the arc $C_{0}$ under consideration.

The problem to be studied in this paper is that of minimizing a function

$$
I(C)=g(a)+\int_{C} f(a, x, y, \dot{y}) d x
$$

in a class of arcs

$$
\text { C: } \quad a^{h}, y^{i}(x) \quad\left(x^{1} \leqq x \leqq x^{2} ; h=1, \cdots, r ; i=1, \cdots, n\right)
$$

satisfying conditions of the form

$$
\phi^{\beta}(a, x, y, \dot{y})=0 \quad(\beta=1, \cdots, m<n),
$$

Presented to the Soc'ety, November 29, 1946; received by the editors December 10, 1946.

(1) Numbers in brackets refer to the references cited at the end of the paper. 


$$
x^{s}=X^{s}(a), \quad y^{i}\left(x^{s}\right)=Y^{i s}(a)
$$

The components $a^{h}$ of $C$ are.independent of $x$. It will be shown in $\$ 8$ below that if $C_{0}$ is an arc satisfying conditions (1.1) and (1.2) and the conditions described in $\$ 2$ there is a neighborhood $\mathfrak{F}$ of $C_{0}$ and a constant $\rho>0$ such that the inequality

$$
I(C)-I\left(C_{0}\right) \geqq \rho K\left(C, C_{0}\right)
$$

holds for every admissible arc in $\mathfrak{F}$ satisfying the conditions (1.1) and (1.2). Here

$$
\begin{aligned}
K\left(C, C_{0}\right)= & \left(a^{h}-a_{0}^{h}\right)\left(a^{h}-a_{0}^{h}\right) \\
& +\int_{C}\left\{\left[1+\left(\dot{y}^{i}-\dot{y}_{0}^{i}\right)\left(\dot{y}^{i}-\dot{y}_{0}^{i}\right)\right]^{1 / 2}-1\right\} d x
\end{aligned}
$$

and $a_{0}, y_{0}^{\mathbf{8}}(x)$ are the functions defining $C_{0}$, extended if necessary so that the integral (1.4) is well defined. As was remarked above, the integral (1.4) can be considered a measure of the deviation of the arc $C$ from $C_{0}$, provided $C$ satisfies the end conditions (1.2). Further inequalities of the form (1.3) are also given in $\$ 8$.

One of the interesting features of the sufficiency proof given below is that the problem is reduced in $\S \S 3,4$ to the study of one in which there are no differential side conditions (cf. [8]). The problem so obtained is of a type which, as far as we know, has not been treated heretofore and to which the usual field theory does not appear to be applicable. An analogue of the theorem of Lindeberg is given in $\$ 5$.

The methods here used are applicable with simple modifications to the case when the arcs $C$ are also required to satisfy isoperametric conditions of the form

$$
I^{\sigma}(C)=g^{\sigma}(a)+\int_{C} f^{\sigma}(a, x, y, \dot{y}) d x=0 \quad(\sigma=1, \cdots, p) .
$$

The modifications necessary are like those made by the author in the parametric case (see [6]) and will be left to the reader.

In carrying out our sufficiency proof it will be convenient at times to assume that the functions $X^{1}(a)$ and $X^{2}(a)$ given in (1.1) and (1.2) are constants. This hypothesis greatly simplifies our computations. Moreover no generality is lost thereby since this situation can be realized by replacing $x$ by a new variable $t$ defined by the equation

$$
x=X^{1}(a)+t\left[X^{2}(a)-X^{1}(a)\right],
$$

provided $X^{2}(a)>X^{1}(a)$, as we shall suppose.

2. A sufficiency theorem. The terminology and notations to be used are 
essentially those used by the author for the parametric case [6]. For example, we shall use the vector notation

$C:$

$$
a, \quad y(x)
$$$$
\left(x^{1} \leqq x \leqq x^{2}\right)
$$

for an $\operatorname{arc} C$. The lengths of vectors $a, y, p, \cdots$ will be denoted by $|a|$, $|y|,|p|, \cdots$. A repeated index in a term will denote summation, except for the indices $q$ and $s$.

It will be assumed that the functions $f(a, x, y, p), \phi^{\beta}(a, x, y, p)$ and their derivatives with respect to $a^{h}, y^{i}, p^{i}$ are continuous and have continuous derivatives on an open set $\Re$ in $(a, x, y, p)$-space. The functions $g(a), X^{s}(a)$, $Y^{i s}(a)$ are assumed to be of class $C^{\prime \prime}$ on $\Re$. We suppose that $X^{1}(a)<X^{2}(a)$ on $\Re$. The subset of $\Re$ on which $\phi^{\beta}=0$ will be denoted by $\mathfrak{D}$.

By an admissible arc $C$ will be meant one for which the functions $y^{i}(x)$ defining $C$ are absolutely continuous and have integrable square derivatives and whose elements $[a, x, y(x), \dot{y}(x)]$ are in $\Re$ for almost all $x$ on $x^{1} x^{2}$. We shall say that $C$ satisfies the differential equations $\phi^{\beta}=0$ if $\phi^{\beta}[a, x, y(x), \dot{y}(x)]=0$ for almost all $x$ on $x^{1} x^{2}$.

We shall be concerned with a particular admissible arc $C_{0}$ :

$$
a_{0}, \quad y_{0}(x)
$$$$
\left(x^{1} \leqq x \leqq x^{2}\right)
$$

of class $C^{\prime \prime}$ that satisfies the conditions (1.1) and (1.2). It will be assumed that the matrix $\left\|\phi_{p i}^{\beta}\right\|$ has rank $m$ along $C_{0}$ and that there exists a set of multipliers, not identically zero,

$$
\lambda^{0} \geqq 0, \quad \lambda^{\beta}(x)
$$

of class $C^{\prime}$ such that if we write

$$
F(a, x, y, p, \lambda)=\lambda^{0} f+\lambda^{\beta} \phi^{\beta}, \quad G(a)=\lambda^{0} g(a)
$$

then the equations

$$
\begin{gathered}
(d / d x) F_{p^{i}}=F_{y^{i}}, \\
G_{h}+\left[\left(F-\dot{y}^{i} F_{p^{b}}\right) X_{h}^{s}+F_{p^{i}} Y_{h}^{i s}\right]_{a-1}^{s-2}+\int_{C_{0}} F_{a^{h}} d x=0
\end{gathered}
$$

hold along $C_{0}$. Here and elsewhere the subscript $h$ on $G(a), X^{s}(a), Y^{i s}(a)$ denotes the derivative of these functions with respect to $a^{h}$ at $a=a_{0}$. The quantity in the brackets is to be evaluated at the initial end point of $C_{0}$ when $s=1$ and at the final end point when $s=2$.

We shall make a further restriction on our choice of multipliers (2.1). To this end let $E_{F}(a, x, y, p, q)$ be the $E$-function

$$
E_{p}=F(a, x, y, q)-F(a, x, y, p)-\left(q^{i}-p^{i}\right) F_{p^{b}}(a, x, y, p)
$$

and denote by $E_{L}(p, q)$ the corresponding $E$-function for the integrand 


$$
L(p)=\left(1+p^{i} p^{i}\right)^{1 / 2}
$$

of the length integral. Recall that $\mathfrak{D}$ is the set of all elements $(a, x, y, p)$ in $\Re$ for which $\phi^{\beta}=0$. It will be assumed that there is a neighborhood $\mathfrak{D}_{0}$ of $\cdot C_{0}$ relative to $\mathfrak{D}$ and a constant $\tau>0$ such that the inequality

$$
E_{F}(a, x, y, p, q) \geqq \tau E_{L}(p, q)
$$

holds whenever $(a, x, y, p)$ is in $\mathfrak{D}_{0}$ and $(a, x, y, q)$ is in $\mathfrak{D}$. This is the condition of $E$-dominance recently introduced by the author. It is equivalent to the strengthened condition of Weierstrass together with the condition of nonsingularity $\left({ }^{2}\right)$. The latter condition states that the determinant

$$
\left|\begin{array}{ll}
F_{p^{i} p^{i}} & \phi_{p^{i}}^{\gamma} \\
\phi_{p^{i}} & 0
\end{array}\right|
$$

is different from zero along $C_{0}$.

If it is possible to choose the multipliers (2.1) so that $\lambda^{0}=1$ we shall suppose that they have been so chosen. Then apart from an arbitrary positive constant the totality of multipliers that satisfy the conditions described above are expressible in the form

$$
\lambda^{0} \geqq 0, \quad \lambda^{\beta}(x, b)=\lambda^{\beta}(x)+\lambda^{\beta \sigma}(x) b^{\sigma}
$$$$
(\sigma=1, \cdots, l)
$$

It is to be understood that we may have $l=0$. Here the functions $\lambda^{\beta \sigma}(x)$ form with $\lambda^{0 \sigma}=0$ a maximal set of linearly independent multipliers of the form $\lambda^{0}=0, \lambda^{\beta}(x)$ with which $C_{0}$ satisfies equations (2.1) and (2.3). If $\lambda^{0}=0$ in (2.4), then $\lambda^{\beta}(x)$ is a linear combination of the set $\lambda^{\beta \sigma}(x)(\sigma=1, \cdots, l)$. The functions $\lambda^{\beta \sigma}(x)$ are of class $C^{\prime}$ and the matrix $\left\|\lambda^{\beta \sigma}(x)\right\|$ has rank $l$ on $x^{1} x^{2}$. In what follows we frequently shall make statements about the parameters $b=\left(b^{1}, \cdots, b^{l}\right)$ appearing in (2.4), thereby tacitly implying that $l \geqq 1$. These statements are to be considered to be vacuously true when $l=0$.

In the following pages we shall be interested only in the multipliers belonging to this family. In view of this fact it is convenient to introduce the notation

$$
H(a, x, y, p, b)=\lambda^{0} f+\lambda^{\beta}(x, b) \phi^{\beta}+\theta(a, x, y, p) \phi^{\beta} \phi^{\beta} .
$$

Here $\theta(a, x, y, p)$ is for the moment an arbitrary function of class $C^{\prime \prime}$ on $\Re$. It is readily verified that the conditions described above as well as the further condition imposed on $C_{0}$ in Theorem 2.1 below is independent of our choice of $\theta$. The reason for its introduction will be made clear in Theorem 2.2 and in the next section.

(2) The proof of these remarks can be found in the author's paper [4] on the Weierstrass $E$-function. This paper deals with the parametric problem. However the hypotheses are such that the results are equally applicable to the nonparametric case. 
The $E$-function for $H$ will be denoted by $E_{H}(a, x, y, p, b, q)$. Let $B$ be the set of all elements $b=\left(b^{1}, \cdots, b^{l}\right)$ for which there is a constant $\tau>0$ and a neighborhood $\mathfrak{D}_{0}$ of $C_{0}$ relative to $\mathfrak{D}$ such that the inequality

$$
E_{H}(a, x, y, p, b, q) \geqq \tau E_{L}(p, q)
$$

holds whenever $(a, x, y, p)$ is in $\mathfrak{D}_{0}$ and $(a, x, y, q)$ is in $\mathfrak{D}$. The set $B$ is an open set. To prove this observe that whenever $(a, x, y, p)$ and $(a, x, y, q)$ are in $\mathfrak{D}$ we have

$$
E_{H}(a, x, y, p, b, q)=E_{F}(a, x, y, p, q)+\lambda^{\beta \sigma} b^{\sigma} E_{\phi \beta}(a, x, y, p, q),
$$

where $E_{\phi \beta}$ is the $E$-function for $\phi^{\beta}$. Moreover, as has been shown by the author $\left[4\right.$, p. 59], there is a constant $\tau_{1}>0$ and a neighborhood $\mathfrak{D}_{1}$ of $C_{0}$ relative to $\mathfrak{D}$ such that the inequality

$$
E_{L}(p, q) \geqq \tau_{1}\left|E_{\phi} \beta(a, x, y, p, q)\right|
$$

holds whenever $(a, x, y, p)$ is in $\mathfrak{D}_{1}$ and $(a, x, y, q)$ is in $\mathfrak{D}$. From these remarks it follows readily that if $(2.6)$ is effective for an element $b_{0}$ with a given constant $\tau$ and neighborhood $\mathfrak{D}_{0}$ in $\mathfrak{D}_{1}$, then it will remain effective on a neighborhood of $b_{0}$ provided $\tau$ is replaced by $\tau / 2$. Consequently $B$ is open, as was to be proved. It is clear that the conditions (2.2) and (2.3) with $F$ replaced by $H$ hold on $C_{0}$. It should also be observed that (2.6) implies (see [4, p. 57]) that the inequality

$$
H_{p^{i} p^{i} \pi^{i} \pi^{j}>0}
$$

holds along $C_{0}$ whenever $b$ is in $B$ and $\pi \neq 0$ satisfies the conditions $\phi_{p^{i}}^{\beta} \pi^{i}=0$.

We next introduce the function

$$
J(C, b)=G(a)+\int_{C} H(a, x, y, \dot{y}, b) d x
$$

and observe that $J(C, b)=\lambda^{0} I(C)$ whenever the equation $\phi^{\beta}=0$ holds along $C$. The second variation $J_{2}(\gamma, b)$ of $J(C, b)$ along $C_{0}$ takes the form

$$
J_{2}(\gamma, b)=2 Q(\alpha)+\int_{x^{1}}^{x^{2}} 2 \Omega(\alpha, x, \eta, \dot{\eta}, b) d x .
$$

Here $2 \Omega(\alpha, x, \eta, \pi, b)$ is the second differential of $H(a, x, y, p, b)$ on $C_{0}$ with respect to the variables $a^{h}, y^{i}, p^{i}$, The function $2 Q(\alpha)$ is a quadratic form in $\alpha=\left(\alpha^{1}, \cdots, \alpha^{r}\right)$ such that the coefficient of $\alpha^{h} \alpha^{k}$ is

$$
\begin{aligned}
G_{h k}+ & {\left[\left(H-\dot{y}^{i} H_{p^{i}}\right) X_{h k}^{s}+H_{p^{i}} Y_{h k}^{i s}\right]_{s=1}^{s=2} } \\
& +\left[\left(H_{x}-\dot{y}^{i} H_{y^{i}}\right) X_{h}^{s} X_{k}^{s}+H_{y^{i}}\left(X_{h}^{8} Y_{k}^{i s}+X_{k}^{s} Y_{h}^{i s}\right)+H_{a^{h}} X_{k}^{s}+H_{a^{k}} X_{h}^{s}\right]_{s=1}^{s=2} .
\end{aligned}
$$

As before the subscripts $h, k$ denote derivatives with respect to $a^{h}$ and $a^{k}$ 
at $a=a_{0}$. When $s=1$ the quantity in the brackets is to be evaluated at the initial point of $C_{0}$ and when $s=2$ at the final end point of $C_{0}$. If $X^{1}(a)$ and $X^{2}(a)$ are constant this coefficient takes the simpler form

$$
G_{h k}+\left[H_{p^{s}} Y_{h k}^{i s}\right]_{s=1}^{a-2} \text {. }
$$

The symbol $\boldsymbol{\gamma}$ denotes a set of constants and absolutely continuous functions $\gamma: \quad \alpha^{h}, \eta^{i}(x) \quad\left(x^{1} \leqq x \leqq x^{2} ; h=1, \cdots, r ; i=1, \cdots, n\right)$ whose derivates $\dot{\eta}^{i}(x)$ are integrable square on $x^{1} x^{2}$. Such a system will be called an admissible variation. We shall be interested in admissible variations $\boldsymbol{\gamma}$ that satisfy along $C_{0}$ the differential equations

$$
\Phi^{\beta}(\alpha, x, \eta, \dot{\eta})=\phi_{a^{h} \alpha}^{\beta}{ }^{h}+\phi_{y^{i} \eta}^{\beta}{ }^{i}+\phi_{p^{i} \dot{\eta}}^{\beta}{ }^{i}=0
$$

for almost all $x$ on $x^{1} x^{2}$ and the end conditions

$$
\eta^{i}\left(x^{s}\right)=\left(Y_{h}^{i s}-\dot{y}^{i}(x) X_{h}^{s}\right) \alpha^{h} \quad(s=1,2) .
$$

Again if $X^{1}(a)$ and $X^{2}(a)$ are constants, these equations take the simpler form

$$
\eta^{i}\left(x^{s}\right)=Y_{h}^{i s} \alpha^{h} .
$$

As a first sufficiency theorem we have:

TheOREM 2.1. Let $C_{0}$ have the properties described above. Suppose further that for every non-null admissible variation $\gamma$ satisfying the conditions (2.9) and (2.10) there is an element $b$ in $B$ such that the inequality $J_{2}(\gamma, b)>0$ holds. Then there is a neighborhood $\mathfrak{F}$ of $C_{0}$ in axy-space such that the inequality $I(C)>I\left(C_{0}\right)$ holds for every admissible arc $C \neq C_{0}$ in $\mathfrak{F}$ satisfying the differential equations (1.1) and end conditions (1.2).

This result, stated in somewhat different form, was conjectured by McShane [2, p. 346]. As was stated in the introduction it is a corollary of the corresponding result for the parametric case. The hypothesis used by the author appears to be weaker. However they are equivalent. In this theorem the function $\theta(a, x, y, p)$ appearing in the definition of $J(C, b)$ is of no consequence. The function $\theta$ can be used to establish a new proof of Theorem 2.1 by showing that the following more general result is true.

TheOREM 2.2. Let $C_{0}$ have the properties described in Theorem 2.1. The function $\theta(a, x, y, p)$ used in the definition of the function $J(C, b)$ can be chosen so that there is a neighborhood $\mathfrak{F}$ of $C_{0}$ in axy-space such that given an admissible arc $C \neq C_{0}$ in $\mathfrak{F}$ satisfying the end conditions (1.2) there exists an element $b$ in $B$ such that $J(C, b)>J\left(C_{0}, b\right)$. 
In this theorem we do not require the arc $C$ to satisfy the differential equations (1.1). It is clear that Theorem 2.2 implies Theorem 2.1 in case $\lambda^{0}=1$. If $\lambda^{0}=0$ then $\dot{J}(C, b)=J\left(C_{0}, b\right)=0$ for every arc $C$ satisfying the conditions $\phi^{\beta}=0$. Hence $C_{0}$ is the only arc in $\mathfrak{F}$ satisfying the conditions (1.1) and (1.2). The conclusion in Theorem 2.1 is therefore vacuously true and Theorem 2.1 holds in either case.

3. Preliminary lemmas. The selection of the function $\theta(a, x, y, p)$ appearing in the definition of $H$ will be made in the next section. In doing so we shall make use of certain results which we shall now establish. We begin with the following lemma.

Lemma 3.1. Under the hypothesis of Theorem 2.1, there is for each element $b$ in $B$ a constant $\theta_{b}$ such that if $\theta \geqq \theta_{b}$ the inequality

$$
H_{p^{i} p^{i} \pi^{i} \pi^{j}>0}
$$

holds along $C_{0}$ whenever $\pi \neq 0$. Moreover if

$\gamma_{q}:$

$$
\alpha_{q}, \eta_{q}(x)
$$$$
\left(x^{1} \leqq x \leqq x^{2} ; q=1,2, \cdots\right)
$$

is a set of admissible variations converging uniformly on $x^{1} x^{2}$ to a variation

$\gamma_{0}$ :

$\alpha_{0}, \quad \eta_{0}(x)$

$\left(x^{1} \leqq x \leqq x^{2}\right)$

then

$$
\lim _{q=\infty} \inf J_{2}\left(\gamma_{q}, b\right) \geqq J_{2}\left(\gamma_{0}, b\right)
$$

provided $b$ is in $B$ and $\theta \geqq \theta_{b}$.

The first statement has been established by Reid [1, p. 679] and the author $[4$, p. 57]. The second follows from the fact that when (3.1) holds the function $J_{2}(\gamma, b)$ is lower semi-continuous (see [2, p. 358]).

Lemma 3.2. Under the hypotheses of Theorem 2.1 there is a compact subset $B_{0}$ of $B$ and $a$ constant $\theta_{0}$ such that if $\theta(a, x, y, p) \geqq \theta_{0}$ and $\gamma$ is a non-null admissible variation satisfying the end conditions (2.10), there is an element $b$ in $B_{0}$ such that $J_{2}(\gamma, b)>0$. Moreover the constant $\theta_{0}$ can be chosen so that the inequality (3.1) holds along $C_{0}$ whenever $b$ is in $B_{0}$ and $\pi \neq 0$, provided $\theta \geqq \theta_{0}$.

In this lemma we do not require the variation $\gamma$ to satisfy the differential equations (2.9). In order to prove this result let $P(\gamma, b)$ be the function obtained from $J_{2}(\gamma, b)$ by setting $\theta=0$. Since $\phi^{\beta}=0$ along $C_{0}$ we have

$$
J_{2}(\gamma, b)=P(\gamma, b)+2 \int_{x^{1}}^{x^{2}} \theta \Phi^{\beta} \Phi^{\beta} d x
$$

where $\Phi^{\beta}$ is given by (2.9). Consequently if we set 


$$
Q(\gamma)=2 \int_{x^{1}}^{x^{2}} \Phi^{\beta} \Phi^{\beta} d x
$$

we have

$$
J_{2}(\gamma, b) \geqq P(\gamma, b)+\theta_{0} Q(\gamma)
$$

whenever $\theta \geqq \theta_{0}$, the equality holding when $\theta=\theta_{0}$.

Since $B$ is an open set it is the union of a denumerable set of compact sets $B_{1}, B_{2}, \cdots$ such that $B_{q} \subset B_{q+1}$. If the first statement of the lemma were false there would exist for every integer $q$ a non-null admissible variation

$\gamma_{q}:$

$$
\alpha_{q}^{h}, \quad \eta_{q}^{i}(x)
$$$$
\left(x^{1} \leqq x \leqq x^{2}\right)
$$

satisfying the end conditions (2.10) such that the inequality

$$
P\left(\gamma_{q}, b\right)+q Q\left(\gamma_{q}\right) \leqq J_{2}\left(\gamma_{q}, b\right) \leqq 0
$$

holds for every element $b$ in $B_{q}$. Since the functions at hand are homogeneous in $\gamma$ we can suppose that $\gamma_{q}$ has been chosen so that

$$
\left|\alpha_{q}\right|^{2}+\int_{x^{1}}^{x^{2}}\left\{\left|\eta_{q}(x)\right|^{2}+\left|\dot{\eta}_{q}(x)\right|^{2}\right\} d x=1,
$$

where a pair of vertical bars denotes the length of the vector at hand. As has been shown by McShane [2, pp. 353-355, 375] we can replace the sequence $\left\{\gamma_{q}\right\}$ by a subsequence, which we take to be the original sequence, which converges to a variation

$\gamma_{0}$ :

$$
\alpha_{0}^{k}, \quad \eta_{0}^{i}(x)
$$$$
\left(x^{1} \leqq x \leqq x^{2}\right)
$$

in the sense that

$$
\lim _{q=\infty} \alpha_{q}^{h}=\alpha_{0}^{h}, \quad \lim _{q=\infty} \eta_{q}^{i}(x)=i_{\eta_{0}}^{i}(x) \quad \text { uniformly on } x^{1} x^{2} .
$$

This variation obviously satisfies the end conditions (2.10).

As a next step we shall show that $\gamma_{0}$ satisfies the differential equations (2.9). To this end consider an element $b$ in $B$ and let $q_{b}$ be chosen so that $b$ is in $B_{q}$ whenever $q \geqq q_{b}$. Using Lemma 3.1, we see that there is a constant $\theta_{b}$ such that

$$
\lim _{\boldsymbol{q}=\infty} \inf \left[P\left(\boldsymbol{\gamma}_{\boldsymbol{q}}, \boldsymbol{b}\right)+\theta_{b} Q\left(\boldsymbol{\gamma}_{\boldsymbol{q}}\right)\right] \geqq P\left(\boldsymbol{\gamma}_{0}, b\right)+\theta_{b} Q\left(\boldsymbol{\gamma}_{0}\right) .
$$

Using the inequality (3.3), which holds when $q \geqq q_{b}$, together with the relations (3.6) and $Q(\gamma) \geqq 0$, we see that

$$
\lim _{q=\infty} \inf Q\left(\gamma_{q}\right) \leqq 0 .
$$


But since the Legendre condition holds for $Q(\gamma)$ we have (see $[2$, p. 358])

$$
\underset{\boldsymbol{c}=\infty}{\lim \inf } Q\left(\gamma_{\boldsymbol{q}}\right) \geqq Q\left(\gamma_{0}\right) \geqq 0 .
$$

Consequently $Q\left(\gamma_{0}\right)=0$. From the definition of $Q$ it is clear that this can be the case only if $\gamma_{0}$ satisfies the differential equations $\Phi^{\beta}=0$ for almost all values of $x$ on $x^{1} x^{2}$, as was to be proved.

We shall show next that $\gamma_{0}$ must be the null variation. Suppose this were not the case. Then by our hypotheses there is an element $b$ in $B$ such that $J_{2}\left(\gamma_{0}, b\right)>0$. Using this value of $b$ in the last paragraph it is seen by (3.6), with $Q\left(\gamma_{0}\right)=0$ and $P\left(\gamma_{0}, b\right)>0$, that for large values of $q$ one has

$$
P\left(\gamma_{q}, b\right)+\theta_{b} Q\left(\gamma_{q}\right)>0,
$$

contradicting the inequality (3.3) which also holds for large values of $q$. It follows that $\gamma_{0}$ is the null variation.

We shall complete the proof of the lemma by showing that $\gamma_{0}$ cannot be the null variation. For suppose that this is the case. Let $b$ be an element in $B$ and take $\theta=\theta_{b}$, where $\theta_{b}$ is chosen as described in Lemma 3.1. Then by virtue of (3.6) we have

$$
\liminf _{\boldsymbol{q}=\infty} J_{2}\left(\gamma_{q}, b\right)=\liminf _{\boldsymbol{q}=\infty}\left[P\left(\gamma_{q}, b\right)+\theta_{b} Q\left(\gamma_{q}\right)\right] \geqq 0
$$

since $P\left(\gamma_{0}, b\right)=Q\left(\gamma_{0}\right)=0$. Using (3.3), we see that the equality must hold. Consequently, by virtue of (3.5) with $\alpha_{0}^{h}=\eta_{0}^{3}=0$ and the definition of $J_{2}(\gamma, b)$ we have

$$
0=\liminf _{q=\infty} J_{2}\left(\gamma_{q}, b\right)=\liminf _{Q=\infty} \int_{x^{1}}^{x^{2}} H_{p^{b} p^{p} \dot{\eta}_{q}^{j} \dot{\eta}_{q}^{i} d x}
$$

Since by Lemma 3.1 the last integrand is a positive definite form there is a constant $c>0$ such that inequality

$$
H_{p^{i} p^{i} \pi^{i} \pi^{i}} \geqq c \pi^{i} \pi^{i} \geqq c|\pi|^{2}
$$

holds. Consequently equation (3.7) implies that

$$
\liminf _{Q=\infty} \int_{x^{1}}^{x^{2}}\left|\dot{\eta}_{q}\right|^{2} d x=0 .
$$

Using (3.4) and (3.5) we see that

$$
\lim _{q=\infty} \int_{x^{1}}^{x^{2}}\left|\dot{\eta}_{q}\right|^{2} d x=1 .
$$

This contradiction completes the proof of the first statement of Lemma 3.2.

In order to complete the proof of Lemma 3.2 observe that by continuity considerations it is seen that if $\theta=\theta_{b}$ is effective as described in Lemma 3.1 
for an element $b=b_{0}$ it is effective for a neighboring element $b$. Fience by the Heine-Borel theorem there is a value $\theta_{1}$ such that the inequality (3.1) holds for every element $b$ in $B_{0}$ provided $\theta \geqq \theta_{1}$ and $\pi \neq 0$. Increase $\theta_{0}$ if necessary so that $\theta_{0} \geqq \theta_{1}$. The last statement in Lemma 3.2 is then valid, as was to be proved.

In the proof of Theorem 8.1 below we shall use the result given in the following lemma.

Lemma 3.3. Let $\theta_{0}$ and $B_{0}$ be chosen as described in Lemma 3.2 and let

$$
J_{2}(\gamma, b, \sigma)=J_{2}(\gamma, b)+\sigma I_{2}(\gamma, b),
$$

where $I_{2}(\gamma, b)$ is a function of $\gamma, b$ of the same form as $J_{2}(\gamma, b)$. There exists a number $\sigma_{0}>0$ such that given a non-null admissible variation $\gamma$ satisfying the end conditions (2.10), there is an element $b$ in $B_{0}$ such that the inequality

$$
J_{2}(\gamma, b, \sigma)>0
$$

holds whenever $|\sigma|<\sigma_{0}$.

Observe first that since $I_{2}(\gamma, b)$ is of the same form as $J_{2}(\gamma, b)$ there is a constant $\tau$ such that

$$
\left|I_{2}(\gamma, b)\right| \leqq \tau \int|\alpha|^{2}+\int_{x^{1}}^{x^{2}}\left\{|\eta|^{2}+|\dot{\eta}|^{2}\right\} d x
$$

whenever $b$ is in $B_{0}$. Suppose now the lemma is false. Then there exists for every integer $q$ a value $\sigma_{q}$, not exceeding $1 / q$ in absolute value, and a nonnull admissible variation

$\gamma_{q}:$

$$
\alpha_{q}, \quad \eta_{q}(x)
$$$$
\left(x^{1} \leqq x \leqq x^{2}\right)
$$

such that

$$
J_{2}\left(\gamma_{q}, b, \sigma_{q}\right) \leqq 0
$$

whenever $b$ is in $B_{0}$. We can suppose that $\gamma_{q}$ satisfies condition (3.4). Then $\left|I_{2}\left(\gamma_{q}, b\right)\right| \leqq \tau$ by $(3.10)$. Since $\lim _{q-\infty} \sigma_{q}=0$ we have

$$
\liminf _{q=\infty} J_{2}\left(\gamma_{q}, b, \sigma_{q}\right)=\liminf _{q=\infty} J_{2}\left(\gamma_{q}, b\right) \leqq 0
$$

for every $b$ in $B_{0}$. As in the proof of Lemma 3.2 we can replace the sequence $\left\{\gamma_{q}\right\}$ by a subsequence, again denoted by $\left\{\gamma_{q}\right\}$, which converges uniformly on $x^{1} x^{2}$ to an admissible variation $\gamma_{0}$ satisfying the end conditions (2.10). By (3.11) and (3.2), which holds because of our choice of $\theta_{0}$, we have $J_{2}\left(\gamma_{0}, b\right) \leqq 0$ for every element $b$ in $B_{0}$. It follows from the last lemma that $\gamma_{0}$ is null. Hence equation (3.7) holds. As was seen in the proof of Lemma 3.2 this is impossible. This contradiction establishes Lemma 3.3.

4. Choice of the function $\theta(a, x, y, p)$. We are now in position to select 
a function $\theta$ that will be effective as described in Theorem 2.2. This choice will be made in the following:

Lemma 4.1. Let $C_{0}$ satisfy the conditions described in Theorem 2.1 and select a compact set $B_{0}$ of $B$ and a constant $\theta_{0}$ having the properties described in Lemma 3.2. There exists a function $\theta(a, x, y, p) \geqq \theta_{0}$ of class $C^{\prime \prime}$ such that when this function is used in the definition (2.5) of $H(a, x, y, p, b)$ there is a neighborhood $\Re_{0}$ of $C_{0}$ relative to $\Re$ and a constant $\tau>0$ such that the inequality

$$
E_{H}(a, x, y, p, b, q) \geqq \tau E_{L}(p, q)
$$

holds whenever $b$ is in $B_{0},(a, x, y, p)$ is in $\Re_{0}$ and $(a, x, y, q)$ is in $\Re$.

The proof of this result is like that used by the author $[4, \S 8]$ and Miss Lewis $[8, \S 4]$ in a similar situation. Select $\theta=\theta_{0}$ in $H$. We first observe that there is a constant $\tau_{1}>0$ and a neighborhood $\Re_{1}$ of $C_{0}$ such that the inequality

$$
E_{H}(a, x, y, p, b, q) \geqq \tau_{1} E_{L}(p, q)
$$

holds whenever $b$ is in $B_{0},(a, x, y, q)$ is in $\mathfrak{D}$ and $(a, x, y, p)$ is in the intersection of $\mathfrak{D}$ and $\Re_{1}$. For by the argument like that following (2.6) it is seen for each element $b_{0}$ in $B_{0}$ the constant $\tau_{1}$ and the neighborhood $\Re_{1}$ can be chosen so as to be effective for neighboring elements $b$. It follows from the Heine-Borel theorem and the compactness of $B_{0}$ that $\Re_{1}$ and $\tau_{1}$ can be chosen so as to be effective for every $b$ in $B_{0}$, as described above. By virtue of Taylor's theorem and the continuity of our functions it follows from (3.1) that we can diminish $\tau_{1}$ and $\Re_{1}$ so that the inequality (4.2) also holds whenever $b$ is in $B_{0}$ and $(a, x, y, p),(a, x, y, q)$ are in $\Re_{1}$ but not necessarily in $\mathfrak{D}$.

We shall show next that a constant $\tau$ can be chosen so that there exists a second neighborhood $\Re_{0}$ of $\Re_{\mathrm{i}}$ whose closure is interior to $\Re_{1}$ such that the inequality (4.1) holds whenever $(a, x, y, p)$ is in $\Re_{0},(a, x, y, q)$ is in $\mathfrak{D}$ and $b$ is in $B_{0}$. To this end we select first a neighborhood $\Re^{*}$ of $C_{0}$ whose closure is in $\Re_{1}$. Then there is a positive constant $\epsilon<1$ such that if $(a, x, y, p)$ is in $\Re^{*}$ and $(a, x, y, q)$ is exterior to $\Re_{1}$ we have

$$
3 \in L(q) \leqq E_{L}(p, q) \leqq 2 L(q) .
$$

We next select $\Re_{0}$ so small that there exist $n$ continuous functions $r^{i}(a, x, y, p)$ defined on $\Re_{0}$ such that if $(a, x, y, p)$ is in $\Re_{0}$, then $(a, x, y, r)$ is in $\Re^{*}$ and $\phi^{\beta}(a, x, y, r)=0$. Here we are using the notation $r^{i}$ for $r^{i}(a, x, y, p)$. We have

$$
E_{H}(a, x, y, p, b, q)=E_{H}(a, x, y, r, b, q)+R
$$

where

$$
\begin{aligned}
R= & q^{i}\left[H_{p^{i}}(a, x, y, r, b)-H_{p^{i}}(a, x, y, p, b)\right] \\
& +\left[p^{i} H_{p^{i}}(a, x, y, p, b)-r^{i} H_{p^{i}}(a, x, y, r, b)\right] \\
& +H(a, x, y, r, b)-H(a, x, y, p, b) .
\end{aligned}
$$


If $\Re_{0}$ is taken sufficiently small we have

$$
|R|<\epsilon \tau_{1} L(q)
$$

for every element $(a, x, y, p)$ in $\Re_{0}$ and $b$ in $B_{0}$. It follows from (4.2) and (4.3) that if $b$ is in $B_{0},(a, x, y, p)$ is in $\Re_{0}$ and $(a, x, y, q)$ is in $\mathcal{D}$ but not in $\Re_{1}$ we have

$$
E_{H}(a, x, y, p, b, q) \geqq \tau_{1} E_{L}(r, q)-\epsilon \tau_{1} L(q) \geqq 2 \epsilon \tau_{1} L(q) \geqq \epsilon \tau_{1} E_{L}(p, q) .
$$

Setting $\tau=\epsilon \tau_{1}$ in (4.1) we obtain the result described at the beginning of this paragraph.

We now select open sets $\Re_{2}, \Re_{3}, \ldots$ whose union is $\Re$ and which have the property that the closure of $\Re_{j}$ is in $\Re_{j+1}$ for $j=1,2, \ldots$. Let $\theta_{j}(a, x, y$, $p$ ) be functions of class $C^{\prime \prime}$ such that

$$
\theta_{j}=0 \text { on } \Re_{j-1}, \quad \theta_{i} \geqq 0 \text { on } \Re_{j}, \quad \theta_{j}=1 \text { on } \Re-\Re_{j} .
$$

In what follows it will be understood that $b$ is in $B_{0}$ and $(a, x, y, p)$ is in $\Re_{0}$. If $(a, x, y, q)$ is in $\Re_{j+1}-\Re_{j}(j \geqq 1)$, then (4.1) holds if $\phi^{\beta}(a, x, y, q)=0$ and hence, by continuity, if

$$
\phi^{\beta}(a, x, y, q) \phi^{\beta}(a, x, y, q)<\epsilon_{j} E_{L}(p, q),
$$

where $\epsilon_{j}$ is a small positive constant. Select a constant $\delta_{j}$ such that

$$
E_{H}(a, x, y, p, b, q)>\delta_{j}
$$

whenever $(a, x, y, q)$ is in $\Re_{j+1}-\Re_{j}$. Let $d_{j}$ be a positive constant such that the inequality

$$
d_{j} \epsilon_{j} E_{L}(p, q)+\delta_{j}>\tau_{1} E_{L}(p, q)
$$

holds on this set. Set

$$
\theta(a, x, y, p)=\theta_{0}+d_{j} \theta_{j} \quad(j=1,2, \cdots ; j \text { summed }) .
$$

We then have by (4.5)

$$
\theta-\theta_{0}=0 \text { on } \Re_{0}, \quad \theta-\theta_{0} \geqq 0 \text { on } \Re, \quad \theta-\theta_{0} \geqq d_{j} \text { on } \Re-\Re_{j} .
$$

Setting $h=\left(\theta-\theta_{0}\right) \phi^{\beta} \phi^{\beta}$ we have $h \equiv 0$ on $\Re_{0}$ and

$$
E_{h}(a, x, y, p, q)=h(a, x, y, q) \geqq 0
$$

provided $(a, x, y, p)$ is in $\Re_{0}$, as we have supposed. Let $H^{*}=H+h$. We then have

$$
E_{H^{*}}(a, x, y, p, b, q)=E_{H}(a, x, y, p, b, q)+h(a, x, y, q)
$$

whenever $(a, x, y, p)$ is in $\Re_{0}$. If $(a, x, y, q)$ is in $\Re_{1}$ then (4.1) holds so that

$$
E_{H^{*}}(a, x, y, p, b, q) \geqq \tau E_{L}(p, q) .
$$


The same is true if $(a, x, y, q)$ is in $\Re_{j+1}-\Re_{j}(j \geqq 1)$ and (4.6) holds. If (4.6) fails to hold, then by $(4.10)$

$$
h(a, x, y, q) \geqq\left(\theta-\theta_{0}\right) \epsilon_{j} E_{L}(p, q) \geqq d_{f_{j}} E_{L}(p, q) \quad \text { ( } j \text { not summed). }
$$

It follows from (4.8) and (4.11) that (4.12) holds in this case also. The function $\theta$ defined by (4.9) accordingly has the properties described in Lemma 4.1.

It is not difficult to show that there is a constant $\sigma>0$ such that

$$
L(p) \geqq \sigma L\left(p-\dot{y}_{0}(x)\right) \quad\left(x^{1} \leqq x \leqq x^{2}\right) .
$$

As has been shown by the author [4, p. 59] this implies the existence of a constant $\tau_{1}$ and a neighborhood $\Re_{0}$ of $C_{0}$ such that the inequality

$$
E_{L}(p, q) \geqq \tau_{1} E_{L}\left(p-\dot{y}_{0}(x), q-\dot{y}_{0}(x)\right)
$$

holds. Hence when (2.6) holds we have

$$
E_{H}(a, x, y, p, b, q) \geqq \tau \tau_{1} E_{L}\left(p-\dot{y}_{0}(x), q-\dot{y}_{0}(x)\right) .
$$

We shall be interested in the particular case when $p=\dot{y}_{0}(x)$. This gives:

LEMma 4.2. Under the hypotheses of Lemma 4.1 there exists a constant $\tau>0$ and $a$ neighborhood $\mathfrak{F}$ of $C_{0}$ in axy-space such that the inequality

$$
E_{H}\left(a, x, y, \dot{y}_{0}(x), b, q\right) \geqq \tau E_{L}\left(0, q-\dot{y}_{0}(x)\right)=\frac{\tau\left|q-\dot{y}_{0}(x)\right|^{2}}{1+L\left(q-\dot{y}_{0}(x)\right)}
$$

holds, whenever $b$ is in $B_{0},(a, x, y)$ is in $\mathfrak{F}$ and $(a, x, y, q)$ is in $\Re$.

5. Theorem of Lindeberg. Before proceeding to the proof of Theorem 2.2 it will be convenient to establish an analogue of the theorem of Lindeberg. This will be done in Theorem 5.2 below. The results here obtained are of interest apart from the application to be made in the next section. In the proof we do not utilize all the properties of $J(C, b)$. In fact we shall use only the property described in the conclusion of Lemma 4.1.

The results obtained below are based on the following lemma.

Lemma 5.1. Let $p(a), M(a, x, y, b), N_{i}(a, x, y, b)$ be continuous functions and let

$$
J^{*}(C, b)=p(a)+\int_{C}\left\{M(a, x, y, b) d x+N_{i}(a, x, y, b) d y^{3}\right\} .
$$

Let $B_{0}$ be the subset of $B$ described in Lemma 4.1. Given a constant $\epsilon>0$ there exists a neighborhood $\mathfrak{F}$ of $C_{0}$ in axy-space such that for every admissible arc $C$ in $\mathfrak{F}$ satisfying the end conditions (1.2) and every element $b$ in $B_{0}$ one has

$$
\left|J^{*}(C, b)-J^{*}\left(C_{0}, b\right)\right| \leqq \epsilon\left[1+E_{H}^{*}(C, b)\right],
$$


where

$$
E_{H}^{*}(C, b)=\int_{C} E_{H}\left[a, x, y, \dot{y}_{0}(x), b, \dot{y}\right] d x .
$$

Using the fact that $B_{0}$ is compact the proof can be made by the argument used by the author [5, pp. 75-78] to establish a similar theorem for the parametric case. In the proof one can assume without loss of generality that $X^{1}(a)$ and $X^{2}(a)$ are constants.

The lower semicontinuity of $J(C, b)$ is given in the following theorem.

THEOREM 5.1. Let $B_{0}$ be the compact subset of $B$ described in Lemma 4.1. Given a constant $\epsilon>0$ there is a neighborhood $\mathfrak{F}$ of $C_{0}$ in axy-space such that the inequality

$$
J(C, b) \geqq J\left(C_{0}, b\right)-\epsilon
$$

holds whenever $b$ is in $B_{0}$ and $C$ is an admissible arc in $\mathfrak{F}$ satisfying the end conditions (1.2).

In order to prove this result let

We then have

$$
\begin{aligned}
J^{*}(C, b)=G(a) & +\int_{c}\left\{H\left(a, x, y, \dot{y}_{0}(x), b\right)\right. \\
& \left.+\left(\dot{y}^{i}-\dot{y}_{0}^{i}(x)\right) H_{p^{i}}\left(a, x, y, \dot{y}_{0}(x), b\right)\right\} d x .
\end{aligned}
$$

$$
J(C, b)=J^{*}(C, b)+E_{H}^{*}(C, b),
$$

where $E_{H}^{*}(C, b)$ is given by (5.2). Using the relation $J^{*}\left(C_{0}, b\right)=J\left(C_{0}, b\right)$ it is seen that

$$
J(C, b)-J\left(C_{0}, b\right)=J^{*}(C, b)-J^{*}\left(C_{0}, b\right)+E_{H}^{*}(C, b) .
$$

Given a positive constant $\epsilon<1$ let $\mathfrak{F}$ be chosen so that (5.1) holds as described in Lemma 5.1. It follows that

$$
J(C, b)-J\left(C_{0}, b\right) \geqq-\epsilon+(1-\epsilon) E_{H}^{*}(C, b) \geqq-\epsilon
$$

whenever $b$ is in $B_{0}$ and $C$ is an admissible arc in $\mathfrak{F}$ satisfying the end conditions (1.2). This proves Theorem 5.1.

We are now in position to prove an analogue of the theorem of Lindeberg. To this end consider a second function

$$
I(C, b)=P(a)+\int_{c} R(a, x, y, \dot{y}, b) d x
$$

where $P(a)$ and $R(a, x, y, p, b)$ have the continuity and differentiability prop- 
erties on $\Re$ prescribed for $G(a)$ and $H(a, x, y, p, b)$. It is assumed that the $E$-function $E_{R}$ for $R$ is such that there is a constant $\tau_{1}>0$ and a neighborhood $\Re_{0}$ of $C_{0}$ in axyp-space such that the inequality

$$
E_{H}(a, x, y, p, b, q) \geqq \tau_{1}\left|E_{R}(a, x, y, p, b, q)\right|
$$

holds whenever $(a, x, y, p)$ is in $\Re_{0},(a, x, y, q)$ is in $\Re$ and $b$ is in the set $B_{0}$ described in Lemma 4.1. The length integral

$$
\int_{C} L(\dot{y}) d x
$$

has this property.

THEOREM 5.2. Let $B_{0}$ be the compact subset of $B$ described in Lemma 4.1. If $I(C, b)$ has the properties described above there is a constant $\rho>0$ such that given a constant $\delta>0$ there is a neighborhood $\mathfrak{F}$ of $C_{0}$ in axy-space such that the inequality

$$
J(C, b)-J\left(C_{0}, b\right) \geqq 2 \rho\left\{\left|I(C, b)-I\left(C_{0}, b\right)\right|-\delta\right\}
$$

holds for every admissible arc $C$ in $\mathfrak{F}$ satisfying the end conditions (1.2) and for every $b$ in $B_{0}$. If $C$ also satisfies the condition

$$
\left|I(C, b)-I\left(C_{0}, b\right)\right| \geqq 2 \delta
$$

then

$$
J(C, b)-J\left(C_{0}, b\right) \geqq \rho\left|I(C, b)-I\left(C_{0}, b\right)\right| .
$$

In order to prove this result choose constants $\tau, \tau_{1}$ and a neighborhood $\Re_{0}$ of $C_{0}$ so that the inequalities (5.8) and (4.1) hold as stated. Choose $\rho=\tau_{1} / 2$. Set

$$
\begin{aligned}
H_{\sigma}(a, x, y, p, b) & =H(a, x, y, p, b)+\sigma R(a, x, y, p, b), \\
G_{\sigma}(a) & =G(a)+\sigma P(a)
\end{aligned}
$$

where $\sigma= \pm \rho$ and consider the function

$$
J(C, b, \sigma)=G_{\sigma}+\int_{C} H_{\sigma} d x=J(C, b)+\sigma I(C, b) .
$$

By (5.7) and (4.1) we have

$$
E_{H \sigma}(a, x, y, p, b, q) \geqq(\tau / 2) E_{L}(p, q)
$$

whenever $(a, x, y, p)$ is in $\Re_{0},(a, x, y, q)$ is in $\Re$ and $b$ is in $B_{0}$. It follows that $J(C, b, \sigma)$ satisfies the conditions upon which the proof of Theorem 5.1 is based. Given a constant $\epsilon>0$ there is accordingly a neighborhood $\mathfrak{F}$ (effective for $\sigma=\rho$ and for $\sigma=-\rho$ ) such that the inequality 


$$
J(C, b, \sigma)-J\left(C_{0}, b, \sigma\right)=J(C, b)-J\left(C_{0}, b\right)+\sigma\left[I(C, b)-I\left(C_{0}, b\right)\right] \geqq-\epsilon
$$

holds whenever $b$ is in $B_{0}$ and $C$ is an admissible arc in $\mathfrak{F}$ satisfying the end conditions (1.2). Taking $\sigma= \pm \rho$ one obtains the inequality (5.9) with $\delta=\epsilon / 2 \rho$.

The last statement in Theorem 5.2 follows at once from (5.9). This proves the theorem.

By virtue of the inequality (4.14) the function

$$
K\left(C, C_{0}\right)=\left|a-a_{0}\right|^{2}+\int_{x^{1}}^{x^{2}}\left\{L\left(\dot{y}(x)-\dot{y}_{0}(x)\right)-1\right\} d x,
$$

considered as a function of $C$ with $C_{0}$ held fast, has the properties prescribed for $I(C, b)$. This integral is a measure of the difference between $C$ and $C_{0}$ and will be used in the next section. As a consequence of the last theorem we have the following corollary.

CoROllary. There exists a constant $\rho>0$ such that given a constant $\sigma>0$ there is a neighborhood $\mathfrak{F}$ of $C_{0}$ in axy-space such that the inequality

$$
J(C, b)-J\left(C_{0}, b\right) \geqq \rho K\left(C, C_{0}\right)
$$

holds for every admissible arc $C$ in $\mathfrak{F}$ satisfying the end conditions (1.2) and the relation

$$
K\left(C, C_{0}\right) \geqq \sigma .
$$

Before closing this section it will be convenient to establish the following result which will be useful in the next section.

THEOREM 5.3. Let

$C_{q}$ :

$$
a_{q}, \quad y_{q}(x) \quad\left(x_{1} \leqq x \leqq x_{2} ; q=1,2, \cdots\right)
$$

be a sequence of admissible arcs satisfying the end conditions (1.2) and having

$$
\lim _{q=\infty} a_{q}=a_{0}, \quad \lim _{q=\infty} y_{q}(x)=y_{0}(x) \quad \text { uniformly on } x^{1} x^{2} .
$$

If for an element $b$ in $B_{0}$

$$
\limsup _{q=\infty} J\left(C_{q}, b\right) \leqq J\left(C_{0}, b\right)
$$

then

$$
\lim _{e=\infty} K\left(C_{q}, C_{0}\right)=0
$$

and

$$
\lim _{q=\infty} \dot{y}_{q}(x)=\dot{y}_{0}(x) \quad \text { in measure on } x^{1} x^{2}
$$


Given a constant $\rho>0$ there is a constant $\delta>0$ and an integer $q_{0}$ such that if $M$ is a subset of $x^{1} x^{2}$ of measure at most $\delta$ and $q \geqq q_{0}$ then

$$
0 \leqq \int_{M} l_{q}(x) d x<\rho
$$

where

$$
l_{q}(x)=1+L\left(\dot{y}_{q}(x)-\dot{y}_{0}(x)\right) .
$$

The relation (5.19) follows from Theorem 5.2 with $I(C, b)=K\left(C, C_{0}\right)$ and (5.20) follows from (5.19). To prove the last statement in the theorem let $\rho$ be a positive constant and choose $q_{0}$ such that when $q \geqq q_{0}$ one has $K\left(C_{q}, C_{0}\right)<\rho / 2$ and hence

$$
\int_{x^{1}}^{x^{2}}\left\{l_{q}(x)-2\right\} d x<\rho / 2 .
$$

Let $\delta=\rho / 4$ and consider a subset $M$ of $x^{1} x^{2}$ of measure at most $\delta$. Since the integrand of (5.23) is nonnegative we have

$$
\int_{M}\left\{l_{g}(x)-2\right\} d x<\rho / 2
$$

and hence

$$
\int_{M} l_{q}(x) d x<2 \delta+\rho / 2=\rho
$$

as was to be proved.

6. The variation $\gamma_{0}$. Theorem 2.2 will be established by showing that if it were false there would exist a non-null variation $\gamma_{0}$ satisfying end conditions (2.10) such that $J_{2}\left(\gamma_{0}, b\right) \leqq 0$ for every element $b$ in $B_{0}$, contrary to the result described in Lemma 3.2. It is understood that the function $\theta(a, x, y, p)$, has been chosen so that Lemmas 3.2 and 4.1 are valid. We assume also that the functions $X^{1}(a)$ and $X^{2}(a)$ are constants.

Suppose now the conclusion in Theorem 2.2 is false. Then given an integer $q$ there exists an admissible arc

$C_{q}: \quad a_{q}, \quad y_{q}(x) \quad\left(x^{1} \leqq x \leqq x^{2}\right)$

in the $(1 / q)$-neighborhood of $C_{0}$ satisfying the end conditions (1.2) and having

$$
J\left(C_{q}, b\right) \leqq J\left(C_{0}, b\right)
$$

whenever $b$ is in $B_{0}$. The arc $C_{q}$ is taken distinct from $C_{0}$. In view of Theorem 
5.3 we have $\lim _{q-\infty} \dot{y}_{q}(x)=\dot{y}_{0}(x)$ in measure. By replacing our sequence by a suitably chosen subsequence we can obtain the relations

$$
\begin{array}{r}
\lim _{q=\infty} a_{q}=a_{0}, \quad \lim _{q=\infty} y_{q}(x)=y_{0}(x) \quad \text { uniformly on } x^{1} x^{2}, \\
\lim _{q=\infty} \dot{y}_{q}(x)=\dot{y}_{0}(x) \quad \text { almost uniformly on } x^{1} x^{2} .
\end{array}
$$

Let $k_{q}$ be the positive number defined by the equation

$$
k_{q}^{2}=K\left(C_{q}, C_{0}\right)=\left|a_{q}-a_{0}\right|^{2}+\int_{x^{1}}^{x^{2}}\left\{L\left(\dot{y}_{q}-\dot{y}_{0}\right)-1\right\} d x
$$

where a pair of vertical bars denotes the length of the vector at hand. Following McShane and Myers we consider the variations

$\gamma_{q}:$

$$
\alpha_{q}=\left(a_{q}-a_{0}\right) / k_{q}, \quad \eta_{q}(x)=\left(y_{q}(x)-y_{0}(x)\right) / k_{q} .
$$

Using the fact that we can replace the original sequence $\left\{C_{q}\right\}$ by a subsequence, it can be brought about that the sequence $\left\{\gamma_{q}\right\}$ will have the properties described in the following lemma.

LEMMA 6.1. The variation $\gamma_{q}$ satisfies the relation

$$
\left|\alpha_{q}\right|^{2}+\int_{x^{1}}^{x^{2}}\left|\dot{\eta}_{q}\right|^{2} / l_{q} d x=1
$$

where

$$
l_{q}(x)=1+L\left(\dot{y}_{q}(x)-\dot{y}_{0}(x)\right) .
$$

Moreover, given a constant $\epsilon>0$ there is a constant $\delta>0$ and an integer $q_{0}$ such that if $q \geqq q_{0}$ and $x_{\sigma}^{\prime} x_{\sigma}^{\prime \prime}(\sigma=1, \cdots, N)$ are $N$ nonoverlapping subintervals of $x^{1} x^{2}$ the sum of whose lengths does not exceed $\delta$, then

$$
\sum_{\sigma=1}^{N}\left|\eta_{q}\left(x_{\sigma}^{\prime \prime}\right)-\eta_{q}\left(x_{\sigma}^{\prime}\right)\right|<\epsilon .
$$

Equation (6.4) follows from (6.3) and the relation

$$
L\left(\dot{y}_{q}-\dot{y}_{0}\right)-1=\left|\dot{y}_{q}-\dot{y}_{0}\right|^{2} / l_{q} \text {. }
$$

In order to prove the second statement in the lemma let $\epsilon$ be a positive constant and set $\rho=\epsilon^{2}$. Choose $\delta<\rho$ and $q_{0}$ related to $\rho$ as described in Theorem 5.3. Let $M$ be a set of nonoverlapping subintervals $x_{\sigma}^{\prime} x_{\sigma}^{\prime \prime}$ $(\sigma=1, \cdots, N)$ of $x^{1} x^{2}$ whose length sum does not exceed $\delta$. Then by Theorem 5.3

$$
\int_{M} l_{Q} d x<\rho=\epsilon^{2} .
$$


But

$$
\sum_{\sigma=1}^{N}\left|\eta_{q}\left(x_{\sigma}^{\prime \prime}\right)-\eta_{q}\left(x_{\sigma}^{\prime}\right)\right|=\sum_{\sigma=1}^{N}\left|\int_{x_{\sigma}^{\prime}}^{x_{\sigma}^{\prime \prime}} \dot{\eta}_{q} d x\right| \leqq \int_{M}\left|\dot{\eta}_{Q}\right| d x .
$$

By the inequality of Schwarz

$$
\int_{M}\left|\dot{\eta}_{q}\right| d x \leqq\left[\int_{M}\left|\dot{\eta}_{q}\right|^{2} / l_{q} d x\right]^{1 / 2}\left[\int_{M} l_{q} d x\right]^{1 / 2} .
$$

Hence

$$
\sum_{\sigma=1}^{N}\left|\eta_{q}\left(x_{\sigma}^{\prime \prime}\right)-\eta_{q}\left(x_{\sigma}^{\prime}\right)\right| \leqq\left[\int_{M}\left|\dot{\eta}_{q}\right|^{2} / l_{q} d x\right]^{1 / 2}\left[\int_{M} l_{q} d x\right]^{1 / 2} \cdot
$$

It follows from (6.4) and (6.7) that (6.6) holds, as was to be proved.

LEMMA 6.2. The sequence of arcs $\left\{C_{q}\right\}$ can be chosen so that there exists an admissible variation

$\gamma_{0}$ :

$$
\alpha_{0}, \quad \eta_{0}(x)
$$$$
\left(x^{1} \leqq x \leqq x^{2}\right)
$$

satisfying the end conditions (2.10) and having

$$
\lim _{q=\infty} \alpha_{q}=\alpha_{0}, \quad \lim _{q=\infty} \eta_{q}(x)=\eta_{0}(x) \quad \text { uniformly on } x^{1} x^{2} .
$$

Moreover for every measurable subset $M$ of $x^{1} x^{2}$ on which $\left\{\dot{y}_{q}(x)\right\}$ converges uniformly to $y_{0}(x)$ one has

$$
\int_{M}\left|\dot{\eta}_{0}\right|^{2} d x \leqq \liminf _{q=\infty} \int_{M}\left|\dot{\eta}_{q}(x)\right|^{2} d x \leqq 2 .
$$

Replace the original sequence $\left\{C_{q}\right\}$ by a subsequence, again denoted by $\left\{C_{q}\right\}$, such that $\left\{\alpha_{a}^{n}\right\}$ converges to a value $\alpha_{0}^{h}$ and $\left\{\eta_{q}^{i}(x)\right\}$ has a finite or an infinite limit on a denumerable dense subset $D$ of $x^{1} x^{2}$. By virtue of (6.4), the quantity $\alpha_{0}^{h}$ is finite and since the arcs $C_{q}$ satisfy the end conditions (1.2) we have $(s=1,2)$

$$
\lim _{q=\infty} \eta_{q}^{i}\left(x^{s}\right)=\lim _{q=\infty}\left[Y^{i s}\left(a_{q}\right)-Y^{i \ell}\left(a_{0}\right)\right] / k_{q}=Y_{h}^{i s} \alpha_{0}^{h},
$$

where $Y_{n}^{i s}$ is the derivative of $Y^{i s}(a)$ with respect to $a^{h}$ at $a=a_{0}$. Consequently $\left\{\eta_{a}(x)\right\}$ converges to a finite value at $x=x^{1}$ and $x=x^{2}$. It follows from the last lemma and the Heine-Borel theorem that the sequence $\left\{\eta_{a}^{8}(x)\right\}$ is bounded and hence converges to a finite value at each point of $D$.

Consider now a constant $\rho>0$ and let $\delta$ and $q_{0}$ be related to $\epsilon=\rho / 3$ as described in the last lemma. By the Heine-Borel theorem the interval $x^{1} x^{2}$ can be covered by a finite number of subintervals of lengths $\delta$ with centers at 
points $x_{1}, \cdots, x_{w}$ in $D$. Increase $q_{0}$ if necessary such that

$$
\left|\eta_{q}\left(x_{i}\right)-\eta_{r}\left(x_{j}\right)\right|<\rho / 3 \quad\left(q \geqq r \geqq q_{0}, j=1, \cdots, w\right) .
$$

Consider a point $x$ on $x^{1} x^{2}$ and choose $j$ so that $\left|x_{j}-x\right|<\delta$, then by Lemma 6.1 with $N=1$ we have

$$
\left|\eta_{q}(x)-\eta_{q}\left(x_{j}\right)\right|<\rho / 3
$$

From these inequalities it follows that

$$
\left|\eta_{q}(x)-\eta_{r}(x)\right| \leqq\left|\eta_{q}(x)-\eta_{q}\left(x_{j}\right)\right|+\left|\eta_{q}\left(x_{j}\right)-\eta_{r}\left(x_{j}\right)\right|+\left|\eta_{r}\left(x_{j}\right)-\eta_{r}(x)\right|<\rho,
$$

provided $q \geqq r \geqq q_{0}$. The sequence $\left\{\eta_{q}(x)\right\}$ therefore converges uniformly to a function $\eta_{0}(x)$. Using this fact it follows from the inequality (6.6) that $\eta_{0}(x)$ is absolutely continuous on $x^{1} x^{2}$. Since $X^{*}(a)$ are assumed to be constants it is seen from (6.11) that $\gamma_{0}$ satisfies the end conditions (2.10).

We shall show next that the functions $\eta_{0}^{\prime}(x)$ have integrable square derivates. Following McShane we subdivide the interval $x^{1} x^{2}$ into $2^{k}$ equal intervals by points

$$
x_{1}=x^{1}<x_{2}<\cdots<x_{2^{k}+1}=x^{2} .
$$

Let $p_{k}^{i}(x)$ be continuous functions which coincide with $\eta_{0}^{i}(x)$ at these points and are linear between them. Then

$$
\int_{x^{1}}^{x^{2}}\left|\dot{p}_{k}\right|^{2} d x=\sum_{\sigma=1}^{2^{k}}\left|\eta_{0}\left(x_{\sigma+1}\right)-\eta_{0}\left(x_{\sigma}\right)\right|^{2} /\left(x_{\sigma+1}-x_{\sigma}\right) .
$$

From the relation (6.8) with $N=1$ we see that

$$
\frac{\left|\eta_{q}\left(x_{\sigma+1}\right)-\eta_{q}\left(x_{\sigma}\right)\right|^{2}}{x_{\sigma+1}-x_{\sigma}} \leqq \int_{x_{\sigma}}^{x_{\sigma+1}} \frac{\left|\dot{\eta}_{q}\right|^{2}}{l_{q}} d x\left[\frac{\iint_{x_{\sigma}}^{x_{\sigma+1}} l_{q} d x}{x_{\sigma+1}-x_{\sigma}}\right] .
$$

But

$$
\lim _{\ell=\infty} \int_{x_{\sigma}}^{x_{\sigma+1}} l_{q} d x=2\left(x_{\sigma+1}-x_{\sigma}\right)
$$

Hence

$$
\sum_{\sigma=1}^{2^{k}} \frac{\left|\eta_{0}\left(x_{\sigma+1}\right)-\eta_{\theta}\left(x_{\sigma}\right)\right|^{2}}{x_{\sigma+1}-x_{\sigma}} \leqq 2 \liminf _{Q=\infty} \int_{x^{1}}^{x^{2}} \frac{\left|\dot{\eta}_{Q}\right|^{2}}{l_{q}(x)} d x \leqq 2 .
$$

It follows from (6.12) that

$$
\liminf _{q=\infty} \int_{x^{1}}^{x^{2}} 2\left|\dot{\eta}_{q}\right|^{2} / l_{q} d x \geqq \int_{x^{1}}^{x^{2}}\left|\dot{p}_{k}\right|^{2} d x
$$

Hence by Fatou's lemma $\left|\dot{\eta}_{0}(x)\right|^{2}$ is integrable and 
$2 \geqq \liminf _{e=\infty} \int_{x^{1}}^{x} 2\left|\dot{\eta}_{q}\right|^{2} / l_{q} d x \geqq \liminf _{e=\infty} \int_{x^{1}}^{x}\left|\dot{p}_{k}\right|^{2} d x \geqq \int_{x^{1}}^{x^{2}}\left|\dot{\eta}_{0}(x)\right|^{2} d x$.

The relation (6.10) follows from this relation and the fact that $l_{q}(x)$ converges uniformly to 2 on every subset $M$ of $x^{1} x^{2}$ on which $\left\{\dot{y}_{q}(x)\right\}$ converges uniformly to $\dot{y}_{0}(x)$.

In the sequel it will be assumed that the sequence $\left\{C_{q}\right\}$ has been chosen as described in the last lemma. As a further result we have:

LemMa 6.3. Let $M$ be a measurable subset of $x^{1} x^{2}$ on which $\left\{\dot{y}_{q}(x)\right\}$ converges uniformly to $\dot{y}_{0}(x)$. If $g(x)$ is integrable together with its square, then

$$
\lim _{Q=\infty} \int_{M} g(x)\left(\eta_{Q}^{i}-\eta_{0}^{i}\right) d x=\lim _{Q=\infty} \int_{M} g(x)\left(\dot{\eta}_{Q}^{i}-\dot{\eta}_{0}^{i}\right) d x=0 .
$$

If in addition $g(x)$ is bounded, this equation holds for every measurable subset $M$ of $x^{1} x^{2}$. If $N_{i r}(x)(r=0,1,2, \cdots)$ are continuous functions of $x$ on $x^{1} x^{2}$ such that

$$
\lim _{\ell=\infty} N_{i q}(x)=N_{i 0}(x)
$$

uniformly on $x^{1} x^{2}$

then

$$
\lim _{q=\infty} \int_{M} N_{i q} \dot{\eta}_{q}^{i} d x=\int_{M} N_{i 0} \dot{\eta}_{0}^{i} d x
$$

for every measurable subset $M$ of $x^{1} x^{2}$.

The proof is a simple modification of a proof of a similat result given by McShane [2, p. 356]. By virtue of the relation

$$
\left|\int_{M} g(x)\left(\eta_{Q}^{i}-\eta_{0}^{i}\right) d x\right| \leqq\left(\max \left|\eta_{Q}-\eta_{0}\right|\right) \int_{M}|g(x)| d x
$$

it follows that the first limit in (6.13) has the value zero.

We consider next the second limit. Let $g(x)$ and $M$ have the properties described in the lemma. We can suppose $g(x)=0$ on the complement of $M$. Let $p(x)$ be a function of class $C^{\prime}$ such that

$$
\int_{x^{1}}^{x^{2}}|g(x)-p(x)|^{2} d x<\epsilon^{2} / 128 .
$$

We shall show first that there exists an integer $q_{0}$ such that

$$
\int_{x^{1}}^{x^{2}}|g(x)-p(x)|^{2} l_{q}(x) d x<\epsilon^{2} / 16 .
$$

To prove this let $P$ be a bound for $|g(x)-p(x)|^{2}$ on $x^{1} x^{2}$ if $g(x)$ is bounded 
and on the complement of $M$ if $g$ is unbounded. By Theorem 5.3 there is a constant $\delta>0$ and an integer $q_{0}$ such that if $q \geqq q_{0}$ the inequality $\int_{M_{1}} l_{q}(x) d x$ $<\epsilon^{2} / 32 P$ holds for every set $M_{1}$ of measure at most $\delta$. Let $M_{1}$ be chosen of measure at most $\delta$ such that $\left\{\dot{y}_{q}(x)\right\}$ converges uniformly to $\dot{y}_{0}(x)$ on its complement $M_{2}$. We can suppose $M_{2} \supset M$ if $g(x)$ is unbounded. Increase $q_{0}$ if necessary so that $l_{q} \leqq 4$ on $M_{2}$ when $q \geqq q_{0}$. We then have

$$
\begin{gathered}
\int_{M_{1}}|g(x)-p(x)|{ }^{2} l_{q} d x \leqq P \int_{M_{1}} l_{q}(x) d x<\epsilon^{2} / 32, \\
\int_{M_{2}}|g(x)-p(x)|^{2} l_{q}(x) d x \leqq 4 \int_{x^{1}}^{x^{2}}|g(x)-p(x)|^{2} d x<\epsilon^{2} / 32 .
\end{gathered}
$$

Hence (6.16) holds when $q \geqq q_{0}$, as stated.

Observe next that by virtue of (6.4), (6.10) and the relation $l_{q} \geqq 2$ we have

$$
\int_{x^{1}}^{x^{2}}\left|\dot{\eta}_{q}-\dot{\eta}_{0}\right|^{2} / l_{q} d x \leqq 2 \int_{x^{1}}^{x^{2}}\left|\dot{\eta}_{q}\right|^{2} / l_{q} d x+2 \int_{x^{1}}^{x^{2}}\left|\dot{\eta}_{0}\right|^{2} / l_{q} d x \leqq 4 .
$$

Using this result together with the inequality $(6.16)$ it is seen by the inequality of Schwarz that

$$
\begin{aligned}
& \left|\int_{x^{1}}^{x^{2}}(g-p)\left(\dot{\eta}_{q}^{i}-\dot{\eta}_{0}^{i}\right) d x\right| \\
& \quad \leqq\left[\int_{x^{1}}^{x^{2}}(g-p)^{2} l_{q} d x\right]^{1 / 2}\left[\int_{x^{1}}^{x^{2}}\left|\dot{\eta}_{q}-\dot{\eta}_{0}\right|^{2} / l_{q} d x\right]^{1 / 2}<\epsilon / 2,
\end{aligned}
$$

provided $q \geqq q_{0}$. Moreover since by an integration by parts

$$
\int_{x^{1}}^{x^{2}} p\left(\dot{\eta}_{q}^{i}-\dot{\eta}_{0}^{i}\right) d x=\left[p\left(\eta_{q}^{i}-\eta_{0}^{i}\right)\right]_{x^{1}}^{x^{2}}-\int_{x^{1}}^{x^{2}} p^{\prime}\left(\eta_{q}^{i}-\eta_{0}^{i}\right) d x
$$

we can increase $q_{0}$ if necessary so that

$$
\left|\int_{x^{1}}^{x^{2}} p\left(\dot{\eta}_{q}^{i}-\dot{\eta}_{0}^{i}\right) d x\right|<\epsilon / 2 \quad\left(q \geqq q_{0}\right) .
$$

Combining this result with (6.17) we have for $q \geqq q_{0}$

$$
\left|\int_{x^{1}}^{x^{2}} g\left(\dot{\eta}_{q}^{i}-\dot{\eta}_{0}^{i}\right) d x\right| \leqq\left|\int_{x^{1}}^{x^{2}} p\left(\dot{\eta}_{q}^{i}-\dot{\eta}_{0}^{i}\right) d x\right|+\left|\int_{x^{1}}^{x^{2}}(g-p)\left(\dot{\eta}_{q}^{i}-\dot{\eta}_{0}^{i}\right) d x\right|<\epsilon .
$$

Since $g$ was taken to be identically zero on the complement of $M$ we have accordingly

$$
\lim _{q=\infty} \int_{x^{1}}^{x^{2}} g\left(\dot{\eta}_{q}^{i}-\dot{\eta}_{0}^{i}\right) d x=\lim _{q=\infty} \int_{M} g\left(\dot{\eta}_{q}^{i}-\dot{\eta}_{0}^{i}\right) d x=0,
$$


as was to be proved.

In order to prove the last statement in the theorem we write

$$
\int_{M} N_{i q} \dot{\eta}_{q}^{i} d x=\int_{x^{1}}^{x^{2}} N_{i 0} \dot{\eta}_{q}^{i} d x+\int_{x^{1}}^{x^{2}}\left(N_{i q}-N_{i 0}\right) \dot{\eta}_{q}^{i} d x
$$

By the second statement in the lemma we have

$$
\lim _{q=\infty} \int_{x^{1}}^{x^{2}} N_{i 0} \dot{\eta}_{q}^{i} d x=\int_{x^{1}}^{x^{2}} N_{i 0} \dot{\eta}^{i} d x
$$

By the inequality of Schwarz and (6.4) we have

$$
\begin{aligned}
\left|\int_{x^{1}}^{x^{2}}\left(N_{i q}-N_{i 0}\right) \dot{\eta}_{q}^{i} d x\right| & \leqq\left[\int_{x^{1}}^{x^{2}}\left|N_{q}-N_{0}\right|{ }^{2} l_{q} d x\right]^{1 / 2}\left[\int_{x^{1}}^{x^{2}}\left|\dot{\eta}_{q}\right|^{2} / l_{q} d x\right]^{1 / 2} \\
& \leqq \max \left|N_{q}-N_{0}\right|\left[\int_{x^{1}}^{x^{2}} l_{q} d x\right]^{1 / 2}
\end{aligned}
$$

It follows that the last term in (6.19) has the limit zero. Using (6.20) it is seen that (6.15) holds as stated.

7. Proof of Theorem 2.2. As the next step in the proof of Theorem 2.2 we shall show that

$$
\text { (7.1) } \lim _{q=\infty} \frac{J^{*}\left(C_{q}, b\right)-J^{*}\left(C_{0}, b\right)}{k_{q}^{2}}=\frac{1}{2} J_{2}\left(\gamma_{0}, b\right)-\frac{1}{2} \int_{x^{1}}^{x^{2}} H_{p^{i} p^{i} \dot{\eta}_{0} \dot{\eta}_{0}^{j}} d x,
$$

where $J^{*}(C, b)$ is given by (5.4), $J_{2}(\gamma, b)$ is the second variation of $J(C, b)$ along $C_{0}$ and the last integral is to be evaluated along $C_{0}$. In order to establish this result we observe that by Taylor's theorem the integrand $H_{q}^{*}$ of $J^{*}\left(C_{q}, b\right)$ can be put in the form

$$
H_{q}^{*}=H+k_{q}\left\{H_{a^{h} \alpha_{q}}{ }^{h}+H_{y^{i} \eta_{q}^{i}}+H_{p^{i} \dot{\eta}_{q}}{ }^{i}\right\}+\left(k_{q}^{2} / 2\right)\left\{M_{q}+\dot{\eta}_{q}^{i} N_{i q}\right\},
$$

where $H$ and its derivatives are to be evaluated along $C_{0}$ and

$$
\begin{array}{ll}
\lim _{q=\infty} M_{q}=2 \Omega\left(\alpha_{0}, x, \eta_{0}(x), 0, b\right) & \text { uniformly on } x^{1} x^{2} \\
\lim _{q=\infty} N_{i q}=2 \Omega_{\pi^{i}}\left(\alpha_{0}, x, \eta_{0}(x), 0, b\right) & \text { uniformly on } x^{1} x^{2} .
\end{array}
$$

Using Lemma 6.3 it is seen that

$$
\begin{aligned}
\lim _{q=\infty} \int_{x^{1}}^{x^{2}}\left\{M_{q}+\dot{\eta}_{q}^{i} N_{i q}\right\} d x \\
\quad=\int_{x^{1}}^{x^{2}}\left\{2 \Omega\left(\alpha_{0}, x, \eta_{0}, \dot{\eta}_{0}, b\right)-H_{p^{i} p^{j} \dot{\eta_{0}} \dot{\dot{\eta}_{0}}}^{\dot{i}}\right\} d x .
\end{aligned}
$$


Similarly

$$
\begin{gathered}
G\left(a_{q}\right)=G\left(a_{0}\right)+k_{q} G_{h} \alpha_{q}^{h}+\left(k_{q}^{2} / 2\right) G_{2 q} \\
y^{i s}\left(a_{q}\right)=y^{i s}\left(a_{0}\right)+k_{q} Y_{h}^{i s} \alpha_{q}^{h}+\left(k_{q}^{2} / 2\right) Y_{q}^{i s}
\end{gathered}
$$

where

$$
\lim _{q=\infty} G_{2 q}=G_{h k} \alpha_{0}^{h k} \alpha_{0}^{k}, \quad \lim _{q=\infty} Y_{q}^{i s}=Y_{h k}^{i s} \alpha_{0}^{h k} \alpha_{0}^{k}
$$

By the us: of the Euler equations (2.2) and the transversality condition (2.3) with $F=H$ we find by (7.2) and (7.4) that

$$
\frac{J^{*}\left(C_{q}, b\right)-J^{*}\left(C_{0}, b\right)}{k_{q}^{2}}=\frac{1}{2}\left\{G_{2 q}+\left[H_{p^{*}} Y_{q}^{i s}\right]_{s-1}^{a-2}+\int_{x^{1}}^{x^{2}}\left\{M_{q}+\dot{\eta}_{q}^{i} N_{i q}\right\} d x\right\} .
$$

In view of (7.3) and (7.5) it follows that the relation (7.1) holds, as was to be proved.

We shall show next that

(7.6) $\liminf _{q=\infty} k_{q}^{-2} \int_{x^{1}}^{x^{2}} E_{H}\left(a_{q}, x, y_{q}, \dot{y}_{0}, b, \dot{y}_{q}\right) d x \geqq \frac{1}{2} \int_{x^{1}}^{x^{2}} H_{p^{q} p^{j} \dot{\eta}_{0} \dot{\eta}_{0}}^{i} d x$,

where the second integral is to be evaluated along $C_{0}$. To this end let $M$ be a subset of $x^{1} x^{2}$ on which the sequence $\left\{\dot{y}_{q}(x)\right\}$ converges uniformly to $\dot{y}_{0}(x)$. Then for large values of $q$ we have, by Taylor's theorem,

$$
E_{H}\left(a_{q}, x, y_{q}, \dot{y}_{0}, b, \dot{y}_{q}\right)=k_{q}^{2} A_{i j_{q}}(x){\dot{\eta_{q}}}_{\dot{\eta}_{q}}^{i} \quad(x \text { on } M),
$$

where

$$
\lim _{q=\infty} A_{i i^{\natural}}(x)=A_{i j}(x)
$$

uniformly on $M$

and

$$
2 A_{i j}=H_{p^{i} p^{i}}\left(a_{0}, x, y_{0}(x), \dot{y}_{0}(x), b\right) .
$$

Consider now the equation

$$
\int_{M} A_{i j q} \dot{\eta}_{q}^{i} \dot{\eta}_{q}^{j} d x=\int_{M} A_{i} \dot{\eta}_{q}^{i} \dot{\eta}_{q} d x+\int_{M}\left(A_{i j q}-A_{i j}\right) \dot{\eta}_{q}^{i} \dot{\eta}_{q}^{j} d x
$$

By virtue of the relations (7.8) and (6.10) the last integral has the limit zero as $q$ becomes infinite. I't follows that

$$
\liminf _{q=\infty} \int_{M} A_{i j q} \dot{\eta}_{q}^{i} \dot{\eta}_{q}^{j} d x=\liminf _{q=\infty} \int_{M} A_{i} \dot{\eta}_{q}^{i} \dot{\eta}_{q}^{j} d x .
$$

By the use of Lemma 6.3 we have 


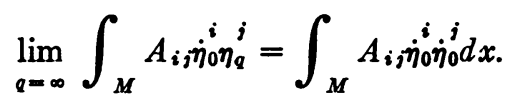

Hence

$$
\liminf _{Q=\infty} \int_{M} A_{i j q} \dot{\eta}_{q}^{i} \dot{\eta}_{q}^{j} d x=\int_{M} A_{i} \dot{\eta}_{\eta_{0}}^{i} \dot{\eta}_{0}^{j}+\liminf _{Q=\infty} \int_{M} A_{i j}\left(\dot{\eta}_{Q}^{i}-\dot{\eta}_{0}^{i}\right)\left(\dot{\eta}_{Q}^{j}-\dot{\eta}_{0}^{j}\right) d x .
$$

The last term is non-negative since, by (7.9) and (3.1), the quadratic form $A_{i j} \pi^{i} \pi^{i}$ is positive definite. It follows from (7.7) and (7.9) that

$$
\liminf _{q=\infty} k_{q}^{-2} \int_{M} E_{H}\left(a_{q}, x, y_{q}, \dot{y}_{0}, b, \dot{y}_{q}\right) d x \geqq 2^{-1} \int_{M} H_{p^{s} p^{j} \dot{\eta}_{0} \dot{\eta}_{0}^{j}}{ }^{j} x
$$

For large values of $q$ we have

$$
E_{H}\left(a_{q}, x, y_{q}(x), \dot{y}_{0}(x), b, \dot{y}_{q}(x)\right) \geqq 0,
$$

except on a set of measure zero. Hence

$$
\liminf _{q=\infty} k_{q}^{-2} E_{H}^{*}\left(C_{q}, b\right) \geqq 2^{-1} \int_{M} H_{p^{i}} \dot{p}_{\dot{\eta}_{0}}^{i} \dot{\eta}_{0}^{j} d x
$$

Since $\left\{\dot{y}_{a}^{8}(x)\right\}$ converges to $\dot{y}_{0}^{t}(x)$ almost uniformly on $x^{1} x^{2}$ it follows from our choice of $M$ and the integrability of $\left|\dot{\eta}_{0}\right|^{2}$ that (7.6) holds, as stated.

Combining (7.6), (7.1), (6.1) and (5.6) it is found that

$$
0 \geqq \liminf _{q=\infty} \frac{J\left(C_{q}, b\right)-J\left(C_{0}, b\right)}{k_{q}^{2}} \geqq \frac{J_{2}\left(\gamma_{0}, b\right)}{2}
$$

for every element $b$ in $B_{0}$. If $\gamma_{0}$ were not null, there would exist, by Lemma 3.2 , an element $b$ in $B_{0}$ such that $J_{2}\left(\gamma_{0}, b\right)>0$. It follows that $\gamma_{0}$ is null. Using (7.1) and (5.6) again, we see from (7.10) that

$$
\underset{q=\infty}{\lim \inf } k_{q}^{-2} E_{H}^{*}\left(C_{q}, b\right) \leqq 0 .
$$

But by (4.13) there is a constant $\tau>0$ such that

$$
0 \geqq \liminf _{q=\infty} \overrightarrow{k q}_{q}^{-2} E_{H}^{*}\left(C_{q}, b\right) \geqq \tau \liminf _{q=\infty} \int_{x^{1}}^{x^{2}}\left|\dot{\eta}_{q}\right|^{2} / l_{q} d x .
$$

This is impossible in view of equation (6.4). This proves Theorem 2.2.

8. Further results. Consider now a second integral $I(C, b)$ having the properties described in the paragraph preceding Theorem 5.2. Suppose in addition that $C_{0}$ satisfies the Euler equations and transversality conditions for $I(C, b)$, whenever $b$ is in $B_{0}$. We can now prove the following theorem.

TheOREM 8.1. If $I(C, b)$ has the properties described above there exists a 
constant $\rho>0$ and a neighborhood $\mathfrak{F}$ of $C_{0}$ such that given an admissible arc $C$ in $\mathfrak{F}$ satisfying the end conditions (1.2), there is an element $b$ in $B_{0}$ such that

$$
J(C, b)-J\left(C_{0}, b\right) \geqq \rho\left|I(C, b)-I\left(C_{0}, b\right)\right| .
$$

As in the proof of Theorem 5.2 we consider the function

$$
J(C, b, \sigma)=J(C, b)+\sigma I(C, b) .
$$

It is clear that $C_{0}$ satisfies the Euler equations and the transversality conditions for $J(C, b, \sigma)$ since it satisfies the corresponding conditions for each of the functions $J(C, b)$ and $I(C, b)$. Moreover by the proof of Theorem 5.2 there is a constant $\rho>0$ and a neighborhood $\Re_{0}$ of $C_{0}$ such that if $|\sigma| \leqq \rho$ the inequality (5.13) holds whenever $b$ is in $B_{0},(a, x, y, p)$ is in $\Re_{0}$ and $(a, x, y, q)$ is in $\Re$.

Consider next the second variation

$$
J_{2}(\gamma, b, \sigma)=J_{2}(\gamma, b)+\sigma I_{2}(\gamma, b) .
$$

By virtue of Lemma 3.3 there is a constant $\sigma_{0}>0$ such that given a non-null variation $\gamma$ satisfying the end conditions (2.10) there is a value $b$ in $B_{0}$ such that $J_{2}(\gamma, b, \sigma)>0$ if $|\sigma| \leqq \sigma_{0}$. Decrease the number $\rho$ chosen above so that $\rho \leqq \sigma_{0}$. For $\sigma= \pm \rho$ the function $J(C, b, \sigma)$ has the properties of $J(C, b)$ upon which the proof of Theorem 2.2 is based. It follows that the conclusion in Theorem 2.2 is valid for $J(C, b, \sigma)$ as well as for $J(C, b)$. Choose a neighborhood $\mathfrak{F}$ effective for $J(C, b, \sigma)$ with $\sigma= \pm \rho$ as described in Theorem 2.2. Then given an admissible arc $C$ in $\mathfrak{F}$ satisfying the end conditions (1.2) there is an element $b$ in $B_{0}$ such that

$$
0 \leqq J(C, b, \pm \rho)-J\left(C_{0}, b, \pm \rho\right)=J\left(C_{0}, b\right)-J(C, b) \pm \rho\left[I(C, b)-I\left(C_{0}, b\right)\right] .
$$

The inequality (8.1) therefore holds as was to be proved.

Observing that the function $K\left(C, C_{0}\right)$ defined by (5.14) has the properties of $I(C, b)$ we obtain the following corollary.

COROLlaRY 1. Under the hypotheses of Theorem 2.1 there exists a constant $\rho>0$ and $a$ neighborhood $\mathfrak{F}$ of $C_{0}$ in axy-space such that given an admissible arc $C$ in $\mathfrak{F}$ satisfying the end conditions (1.2) there is an element $b$ in $B_{0}$ such that

$$
J(C, b)-J\left(C_{0}, b\right) \geqq \rho K\left(C, C_{0}\right) .
$$

Moreover if $C$ satisfies the differential equations (1.1) then

$$
I(C)-I\left(C_{0}\right) \geqq \rho K\left(C, C_{0}\right) .
$$

This result can be considered to be a generalization of the theorem of Osgood (cf. [6, p. 99]). 


\section{REFERENCES}

1. W. T. Reid, Isoperimetric problems of Bolza in nonparametric form, Duke Math. J. vol. 5 (1939) pp. 675-691.

2. E. J. McShane, Sufficient conditions for a weak relative minimum in the problem of Bolza, Trans. Amer. Math. Soc. vol. 52 (1942) pp. 344-379.

3. F. G. Myers, Sufficient conditions for the problem of Lagrange, Duke Math. J. vol. 10 (1943) pp. 73-97.

4. M. R. Hestenes, The Weirstrass E-function in the calculus of variations, Trans. Amer. Math. Soc. vol. 60 (1946) pp. 51-71.

5. - The theorem of Lindeberg in the calculus of variations, Trans. Amer. Math. Soc. vol. 60 (1946) pp. 72-92.

6. - Sufficient conditions for the isoperimetric problem of Bolza in the calculus of variations, Trans. Amer. Math. Soc. vol. 60 (1946) pp. 93-118.

7. - An alternate sufficiency proof for the normal problem of Bolza, Trans. Amer. Math. Soc. vol. 61 (1947) pp. 256-264.

8. A. L. Lewis, Sufficiency proofs for the problem of Bolza in the calculus of variations, Dissertation, The University of Chicago, 1943.

\section{University of Chicago,}

Chicago, Ill. 